\title{
Study on Mandarin Voiced Consonant Airflow and Air-pressure Based on PAS6600
}

\author{
Xiaohui Yang ${ }^{1, a}$, Yonghong $\mathrm{Li}^{2, \mathrm{~b}}$ and Dawa Pengcuo ${ }^{2, \mathrm{c}}$ \\ ${ }^{123}$ (Key Lab of China's National Linguistic Information Technology, Northwest University for \\ Nationalities, Lanzhou, 730030 China,) \\ a547686498@qq.com, blyhweiwei@126.com, dwpc@163.com
}

\begin{abstract}
Keywords: Voiced Consonant, Peak Expiratory Airflow, Peak Air Pressure, Expiratory Volume Analysis
\end{abstract}

\begin{abstract}
Taking mandarin voiced consonant as its study objects, it has used PAS6600 to collect 3 male and female speakers' articulation signals including speech signal, airflow signal and air-pressure signal in order to explore the mechanism of aerodynamic for mandarin voiced consonant. The study found that: 1 ) in the process of pronunciation [m] and [n] both in peak expiratory airflow (PEA) and expiratory volume (EV) is less than the [l] and [r], the peak air pressure (PAP) of [m] and [n] is also less than [l] and [n], namely in the process of pronunciation, peak expiratory airflow, expiratory volume, peak air pressure of mandarin voiced consonant nasals is less than the lateral sound and approximant. 2) Mandarin voiced consonant peak air pressure, peak expiratory airflow and expiratory volume of men are more than women, this is due to generally male lung capacity is greater than female. Apart from Expiratory airflow duration (EAD) nasal [n], there is on difference between men and women. Mandarin Chinese voiced consonant airflow pressure study further describes a voiced consonant pronunciation mechanism, to further distinguish the nasal, lateral sound and approximant.
\end{abstract}

\section{Introduction}

With the development of science and technology in recent years, equipment used in the study of voice is booming, including a lot of medical research of high-tech equipment, air dynamics system (Phonatory Aerodynamic System6600) is one of them. Study of pressure, expiratory airflow change when people speech, called phonatory aerodynamics [1], About experimental phonetics research mainly has the following several consonants: in the 1980s' Wu Zongji did unaspirated/ aspirated difference features experiment of standard Chinese consonants [2], This article reveals the mandarin aspirated and unaspirated sound is not the difference of expiratory airflow and theoretically illustrates the physiological mechanism of affricate. In 2004, Zeng Ting surveyed the flow of air and acoustic analysis of Hunan dialect consonant [n] and [l], the result shows that Hunan dialect [l] and [n] has a tendency to merge [3]. In 2004, Li Jian used electronic Jaw position instrument to study mandarin Chinese consonant of tongue palatal contact situation in the process of pronunciation, experiments prove that in traditional phonetics people rely on intuitive feelings of descriptions of place of articulation is correct [4]. In $2010 \mathrm{Lv}$ Shi liang used phonatory aerodynamic system discussed the plosive and affricate of standard Chinese consonants , finally draw that in pronunciation between a plosive and affricate expiration and expiratory volume has little to do[5]. This paper based on the voiced consonant of mandarin Chinese as the research object, using phonatory aerodynamic system6600 to explore the aerodynamic characteristics of mandarin voiced consonant.

\section{Experimental Description}

\section{Instrument Introduction.}

Phonatory aerodynamic system 6600 (PAS 6600 ), which was produced by KAY. This system is an important experimental instrument of voice physiological analysis. This experiment selected six 
speakers who are college students have standard mandarin pronunciation and there is no history of voice diseases. Recording in sound insulation good recording room, before recording, Explaining the basic principle of experimental equipment and pronunciation training for several times to speakers, to ensure that in the process of pronunciation air flow facemask clings face, which can ensure the precision of the extracted parameters. Required that finish recording a syllable pause a few seconds and then read the next syllable can ensure the stability of airflow pronunciation.

\section{Parameters Introduction.}

Peak Expiratory Airflow ( $\mathrm{Lit} / \mathrm{Sec}$ ) is in the process of pronunciation, the air flow maximum rate of the glottis in per unit time, generally proportional to the open area of the glottis. The greater of glottis area in per unit time the greater the air flow volume, the greater of air flow rate per unit area

Peak Air Pressure ( $\mathrm{cmH} 2 \mathrm{O}$ ) is the peak pressure value in the process of consonant initials, In the process of pronunciation due to the change of consonants pressure value is bigger, the peak pressure determines the nature of a consonant in a large part.

Expiratory Volume ( $\mathrm{L}$ ) is the amount expiratory airflow volume in the process of pronunciation.

Expiratory Airflow Duration ( $\mathrm{Sec}$ ) is the time length of the observed signals on the consonant.

\section{Experimental Analysis}

According to vibration situation of the vocal cord, consonants can be divided into clear consonant and voiced consonant. Mandarin Chinese have 22 consonant in total , which voiced consonants is only five, three nasals [m], [n], [ng], a lateral [l] and a approximant [r]. Experimental phonetics used the PAS6600 air pressure equipment to collect signal, getting the mandarin Chinese voiced consonant of[m], [n], [l], [r] airflow and air pressure parameter table.

Table 1: mandarin Chinese voiced consonant parameter table (average)

\begin{tabular}{|c|c|c|c|c|c|c|c|c|}
\hline \multirow{2}{*}{ parameters } & \multicolumn{2}{|c|}{$\mathbf{m}$} & \multicolumn{2}{|c|}{ l } & \multicolumn{2}{|c|}{$\mathbf{n}$} & \multicolumn{2}{c|}{ r } \\
\cline { 2 - 10 } & male & female & male & female & male & female & male & female \\
\hline EAD (sec) & 1.12 & 0.89 & 1.51 & 1.57 & 1.09 & 0.65 & 1.99 & 1.26 \\
\hline PAP (cm H2O) & 0.91 & 0.60 & 2.88 & 0.81 & 0.75 & 0.71 & 4.24 & 3.85 \\
\hline PEA (Lit/Sec) & 0.28 & 0.14 & 0.43 & 0.22 & 0.25 & 0.11 & 0.65 & 0.39 \\
\hline EVA (L) & 0.21 & 0.07 & 0.39 & 0.16 & 0.19 & 0.07 & 0.49 & 0.17 \\
\hline
\end{tabular}

Figure 1 is mandarin voiced consonant scatterplot of airflow and air pressure, the abscissa axis represents the peak air pressure, the vertical axis represents the peak airflow, four laps up area from left to right respectively is distribution range of voiced consonant [n], [m], [l] and [r]. We can find in figure 3 that the peak air pressure distribution of $[\mathrm{n}]$ is roughly between 0.2 and 1.0, peak airflow distribution range is roughly between 0.06 and 0.21 ; the peak air pressure distribution of [m] is roughly between 1and 1.7, peak airflow distribution range is roughly between 0.15 and 0.26 ; the peak air pressure distribution of [1] is roughly between 1.8 and 3.1, peak airflow distribution range is roughly between 0.18 and 0.36 ; the peak pressure distribution of [r] is roughly between 4.05 and 6.0 , peak airflow distribution range is roughly between 0.42 and 0.71 ; this four voiced consonant is concentrated in the respective areas and from left to right every sound of peak airflow and peak air pressure increase successively. The peak airflow associated with the area of the glottis to open, the greater the peak airflow, the greater the glottis when the voice of the glottis with open area conversely open area is small, In mandarin voiced consonant, the ranks of peak airflow are $[\mathrm{n}]<[\mathrm{m}]$ $<[1]<[r]$, This shows that glottis open area is less than lateral sound and approximant when articulating nasal. the peak air pressure associates with voiced consonant in the mouth of the pressure value before release the resistance, the orders of peak pressure are $[\mathrm{n}]<[\mathrm{m}]<[\mathrm{l}]<[\mathrm{r}]$, this shows that peak air pressure value of mouth are less than the lateral sound and proximate When pronouncing nasals. 


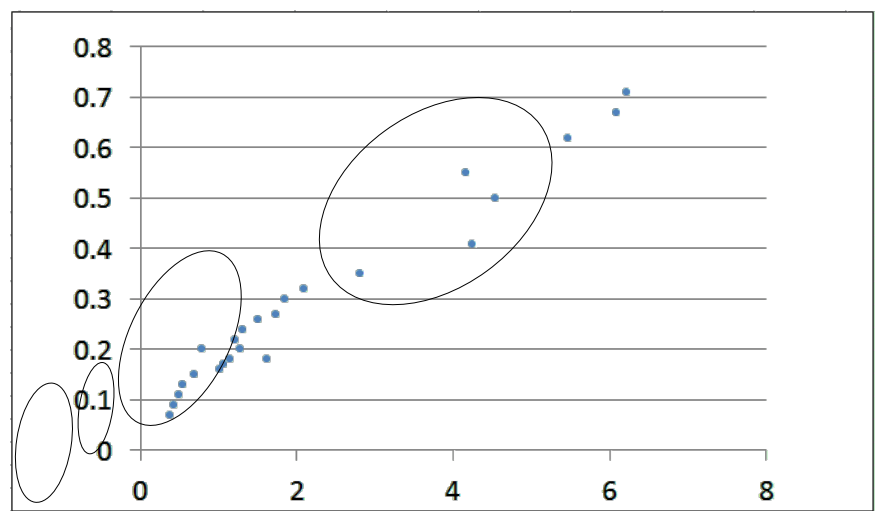

Fig.1 mandarin voiced consonant airflow and air pressure parameters scatterplot

From the manner of articulation, approximant $[\mathrm{r}]$ require the airflow provided by the lungs is biggest, followed by lateral sound [1], finally the nasal [m] and [n]. Air flow volume of men is greater than women, which have close relationship with men lung capacity greater than women.

\section{Conclusion}

According to this experiment we can see that mandarin Chinese voiced consonant airflow pressure study further describes a voiced consonant pronunciation mechanism, to further distinguish the nasal, lateral sound and approximant. Through the experimental analysis also can draw the following conclusion: 1 ) in the process of pronunciation [m] and [n] both in peak expiratory airflow (PEA) and expiratory volume (EV) is less than the [l] and [r], the peak air pressure (PAP) of [m] and [n] is also less than [l] and [n], namely in the process of pronunciation, peak expiratory airflow, expiratory volume, peak air pressure of mandarin voiced consonant nasals is less than the lateral sound and approximant. 2) Mandarin voiced consonant peak air pressure, peak expiratory airflow and expiratory volume of men are more than women, this is due to generally male lung capacity is greater than female. Apart from Expiratory airflow duration (EAD) nasal [n], there is on difference between men and women.

\section{Acknowledgments}

This article is subsidized by Fok Ying Tung Education Foundation (Grant No. 151110)

\section{References}

[1] Lin Tao, Wang li jia. Phonetics profile [M]. Beijing: higher education press, 2014.7

[2] Wu Zong Ji, Talk about "difference characteristics" of the pronunciation of mandarin Chinese and their mutual relations, "Chinese language" in 1980

[3] Zeng Ting, Hunan dialect [n] and [l] airflow and acoustic analysis, the seventh conference on Chinese phonetics and frontier issues of international BBS [J], 2004.

[4] Li Jian, based on the EPG mandarin consonant pronunciation study [D], 2004.2

[5] Lv Shi liang, Research on mandarin plosive and affricate sound air pressure [D], 2012.4 